\title{
Carbon doped GaN buffer layer using propane for high electron mobility transistor applications: Growth and device results
}

\author{
X. Li, ${ }^{1}$ J. Bergsten, ${ }^{2}$ D. Nilsson, ${ }^{1}$ Ö. Danielsson, ${ }^{1}$ H. Pedersen, ${ }^{1}$ N. Rorsman, ${ }^{2}$ E. Janzén, ${ }^{1}$ \\ and U. Forsberg ${ }^{1}$ \\ ${ }^{1}$ Department of Physics, Chemistry, and Biology (IFM), Linköping University, Linköping 58183, Sweden \\ ${ }^{2}$ Microwave Electronics Laboratory, Department of Microtechnology and Nanoscience, \\ Chalmers University of Technology, Göteborg 41296, Sweden
}

(Received 5 October 2015; accepted 29 November 2015; published online 28 December 2015)

\begin{abstract}
The creation of a semi insulating (SI) buffer layer in AlGaN/GaN High Electron Mobility Transistor (HEMT) devices is crucial for preventing a current path beneath the two-dimensional electron gas (2DEG). In this investigation, we evaluate the use of a gaseous carbon gas precursor, propane, for creating a SI GaN buffer layer in a HEMT structure. The carbon doped profile, using propane gas, is a two stepped profile with a high carbon doping $\left(1.5 \times 10^{18} \mathrm{~cm}^{-3}\right)$ epitaxial layer closest to the substrate and a lower doped layer $\left(3 \times 10^{16} \mathrm{~cm}^{-3}\right)$ closest to the 2DEG channel. Secondary Ion Mass Spectrometry measurement shows a uniform incorporation versus depth, and no memory effect from carbon doping can be seen. The high carbon doping $\left(1.5 \times 10^{18} \mathrm{~cm}^{-3}\right)$ does not influence the surface morphology, and a roughness root-mean-square value of $0.43 \mathrm{~nm}$ is obtained from Atomic Force Microscopy. High resolution X-ray diffraction measurements show very sharp peaks and no structural degradation can be seen related to the heavy carbon doped layer. HEMTs are fabricated and show an extremely low drain induced barrier lowering value of $0.1 \mathrm{mV} / \mathrm{V}$, demonstrating an excellent buffer isolation. The carbon doped GaN buffer layer using propane gas is compared to samples using carbon from the trimethylgallium molecule, showing equally low leakage currents, demonstrating the capability of growing highly resistive buffer layers using a gaseous carbon source. (C) 2015 AIP Publishing LLC. [http://dx.doi.org/10.1063/1.4937575]
\end{abstract}

The high output power density and efficiency offered by power amplifiers (PA) based on gallium nitride $(\mathrm{GaN})$ High Electron Mobility Transistors (HEMTs) make them a strong candidate for high frequency and power applications. However, the dispersive behavior of these transistors inhibits large signal performance, which has delayed their adoption in some microwave systems. The dispersive effects are mainly caused by deep electron traps in the buffer and at the surface. These dispersive effects are manifested as a decrease in maximum current (current slump) and on-state conductance (knee walk-out) during large signal operation, leading to lower maximum output power and efficiency. ${ }^{1,2}$

Research on trapping effects in GaN HEMTs has primarily focused on understanding their physical mechanisms and minimizing the current collapse. Earlier, much efforts were dedicated to minimizing effects of surface traps by utilizing different passivation schemes. ${ }^{1-3}$ More recently, there has been a growing interest in the influence of the characteristics of the GaN-buffer on the large-signal performance of GaN HEMTs. The design of the doping profile in the buffer is known to have an effect on trapping phenomena. The buffer in GaN HEMTs is commonly doped with a deep acceptor such as iron (Fe), a deep level acceptor-like impurity, to reduce leakage currents and increase breakdown voltage. ${ }^{4,5}$

From a growth point of view, one of the disadvantages with iron doping is the memory effect during metalorganic chemical vapor deposition (MOCVD) growth. Once the iron containing gas is removed from the growth zone, iron will still be present on the GaN surface, resulting in a slow decrease in the iron incorporation versus thickness. ${ }^{3}$ Therefore, creating abrupt profile using iron doping is a big challenge. An alternative dopant is carbon. Previous investigations using carbon doping have shown promising results, see, for example, Ref. 6. Carbon can be incorporated in two ways: (1) either by tuning the process parameters such as temperature, pressure, and precursor flow rates, to control the decomposition rate of trimethylgallium (TMGa), and thereby also the incorporation of carbon from the resulting methyl groups, or (2) by adding a carbon precursor to the CVD gas mixture.

Our previous investigation on carbon doping using growth process tuning ${ }^{7}$ showed very promising results. It was demonstrated that the carbon profile could be tuned so that the trap density close to the two-dimensional electron gas (2DEG) was reduced, while still demonstrating HEMTs with low leakage currents and small dispersive effects. ${ }^{8}$ However, the growth process tuning involved changes in growth temperature, which can result in poor epitaxial layer uniformity and structural quality, as well as particle down falls during temperature transition.

An alternative approach would be to optimize the growth condition for high quality GaN growth and then add a carbon containing gas to create the semi-insulating (SI) layer. In a recent study, ${ }^{9}$ we identified several potential carbon containing gases that could be used for in-situ carbon doping of GaN.

The purpose of this work is to show the ability to grow tailored carbon doped GaN structure using propane, a gaseous dopant source. Structural and morphological quality is 
evaluated, as well as abruptness and memory effects from carbon doping. HEMTs are fabricated, and the isolation properties of the doped GaN layer using propane is compared with similar GaN HEMT structures where carbon doping is performed using carbon from the TMGa molecule.

A horizontal hot-wall MOCVD reactor was used for the growth of III-nitrides. ${ }^{10} \mathrm{~A}$ (0001) $4 \mathrm{H}-\mathrm{SiC}$ on-axis SI wafer was used for the growth of a HEMT heterostructure. The HEMT structure in this case consists of four different nitride layers: first an AlN nucleation layer followed by a carbon doped GaN buffer layer, an undoped GaN layer, an AlGaN barrier layer, and ended with a GaN cap layer. The nominal $\mathrm{GaN}$ thickness is $1.8 \mu \mathrm{m}, 20 \mathrm{~nm}$ for $\mathrm{AlGaN}$ barrier layer, and $3 \mathrm{~nm}$ for GaN cap layer. The nominal $\mathrm{Al}$ content in the AlGaN layers is typically $20 \%$. The process pressure was 50 mbar during the entire growth. The AlN nucleation layer was grown at $1170^{\circ} \mathrm{C}$ and the remaining GaN/AlGaN/GaN structure was grown at $1040^{\circ} \mathrm{C}$. To improve the wafer uniformity, the substrate was rotated during growth using gas foil rotation. A flow rate of $2.84 \mathrm{ml} / \mathrm{min}(119 \mu \mathrm{mol} / \mathrm{min}) \mathrm{TMGa}$, $0.70 \mathrm{ml} / \mathrm{min}(29 \mu \mathrm{mol} / \mathrm{min})$ of trimethylaluminum (TMAl), and $2000 \mathrm{sccm}$ ammonia $\left(\mathrm{NH}_{3}\right)$ were used as precursors for $\mathrm{Ga}, \mathrm{Al}$, and $\mathrm{N}$, respectively. Propane $\left(\mathrm{C}_{3} \mathrm{H}_{8}\right)$ gas, with a purity of $99.95 \%$, was used as a carbon precursor during the growth of the doped GaN layer.

Normarski differential interference contrast (NDIC) optical microscopy and tapping mode atomic force microscopy (AFM) were used to characterize the surface morphology. The structural property was measured by high resolution X-ray diffraction (HRXRD) with a triple-axis configuration. An interference method ${ }^{11}$ was used to determine the thickness of the epitaxial layers. The as-grown electrical property at room temperature was analyzed by (1) mercury probe Capacitance-Voltage (CV) method; (2) contactless eddy current technique; and (3) contactless Hall technique (LEI 1610 Mobility Measurement System). Secondary Ion Mass Spectrometry (SIMS) measurement was carried out to determine the concentration of $\mathrm{C}, \mathrm{Si}, \mathrm{O}$, and $\mathrm{H}$ throughout the epitaxial layers. The detection limits for this specific analysis were $\mathrm{H}\left[3-5 \times 10^{17} \mathrm{~cm}^{-3}\right], \quad \mathrm{C}\left[5 \times 10^{15} \mathrm{~cm}^{-3}\right]$, O [7-9 $\left.\times 10^{15} \mathrm{~cm}^{-3}\right]$, and Si $\left[0.9-1 \times 10^{16} \mathrm{~cm}^{-3}\right]$, respectively.

HEMT fabrication started with the deposition of a $55 \mathrm{~nm}$ $\mathrm{SiN}_{\mathrm{x}}$ passivation layer in a Low Pressure Chemical Vapor Deposition (LPCVD) system. Mesas were defined through optical lithography and dry etched in a Cl/Ar-plasma. Recessed ohmic contacts were made in an optical lithography process where the recess etching and metal stack were defined with the same resist layer, making it self-aligned. The recess etch was made with a low power $\mathrm{Cl} / \mathrm{Ar}$-plasma, and the metal stack was $\mathrm{Ta} / \mathrm{Al} / \mathrm{Ta}(10 / 280 / 20 \mathrm{~nm}) .{ }^{12-14}$ After annealing at $550{ }^{\circ} \mathrm{C}$, the contact resistance was measured to be 0.33 $\Omega \times \mathrm{mm}$. The gates were defined in a two-step electron-beam lithography process. First, the $0.2 \mu \mathrm{m}$ long gate footprint was etched in the $\mathrm{SiN}_{\mathrm{x}}$-passivation. The second step defined the gate metal stack, Ni/Pt/Au (30/20/400 nm). Contact pads were defined using optical lithography with a Ti/Au metal stack. The source-drain distance was $2.4 \mu \mathrm{m}$, and the gate-drain distance was $1.7 \mu \mathrm{m}$ as measured using a SEM.

The surface of the carbon doped HEMT structure using propane is very smooth. No visible defects could be observed

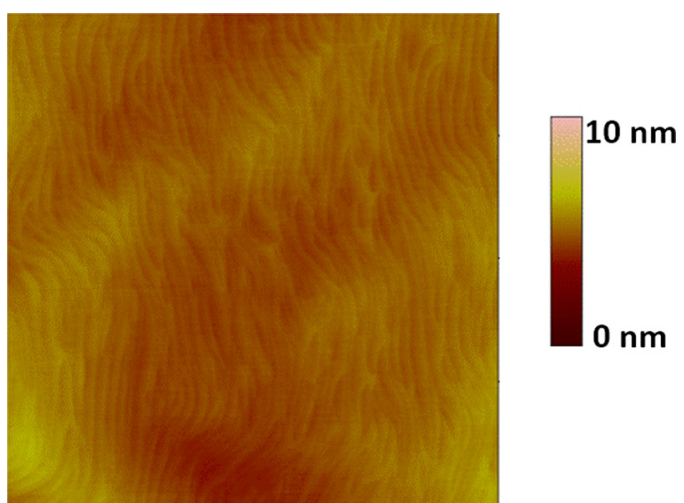

FIG. 1. A $10 \times 10 \mu \mathrm{m}^{2}$ AFM image of the grown HEMT structure.

by NDIC optical microscopy. Fig. 1 shows an AFM image taken over a $10 \times 10 \mu \mathrm{m}^{2}$ area. The surface roughness has a root-mean-square (RMS) value of $0.43 \mathrm{~nm}$. The average thickness of the grown epitaxial layer is $1.92 \mu \mathrm{m} \pm 3 \%$.

HRXRD 2 $\theta-\omega$ curve of the HEMT structure was taken in the vicinity of the GaN (0002) reflection. After simulation using the PANalytical X'Pert Epitaxy software to fit the interference fringes and peaks, it is shown that the AlGaN barrier layer is $23 \mathrm{~nm}$ with an $\mathrm{Al}$ content of $19 \%$ and the GaN cap thickness is around $3 \mathrm{~nm}$. In Fig. 2, the $\omega$ rocking curves of the (102) and (002) reflections are presented, and the full width at half maximum (FWHM) values are 261 arcsec and 200 arcsec, respectively. It is clear that when an optimized process window for GaN is used during growth of the entire carbon doped buffer layer, the structural quality exceeds that when process tuning is used. ${ }^{8}$ This allows the
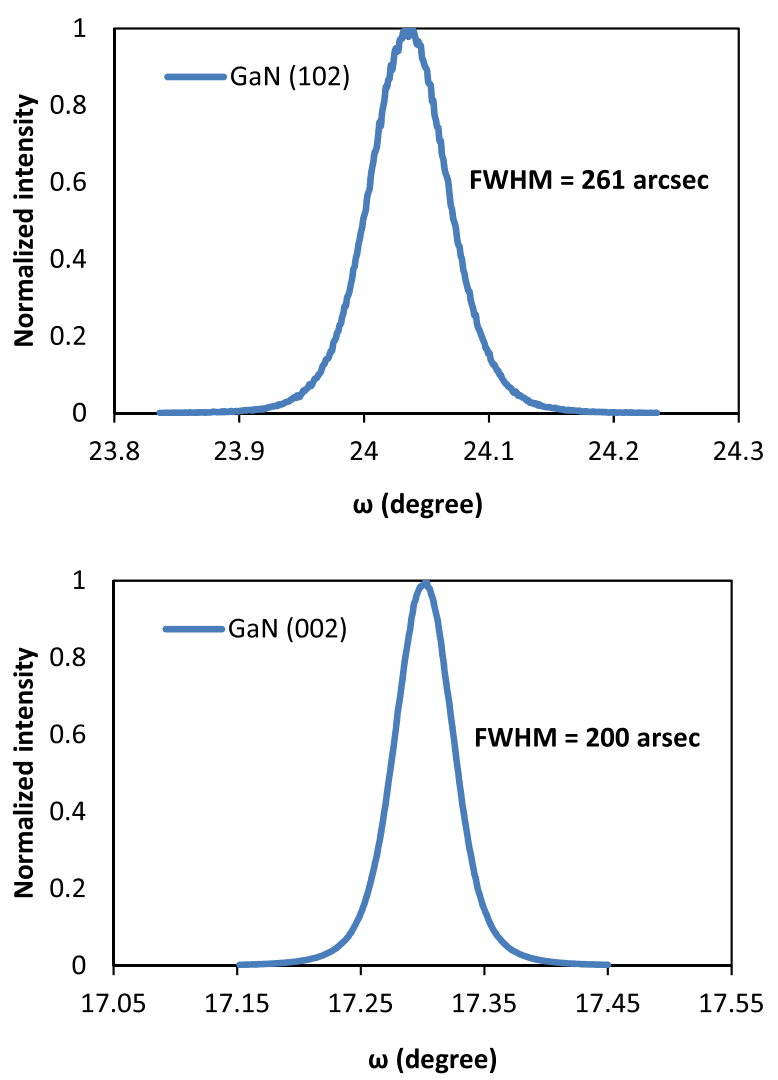

FIG. 2. $\omega$ rocking curve of the (102) and (002) GaN reflection plane. 


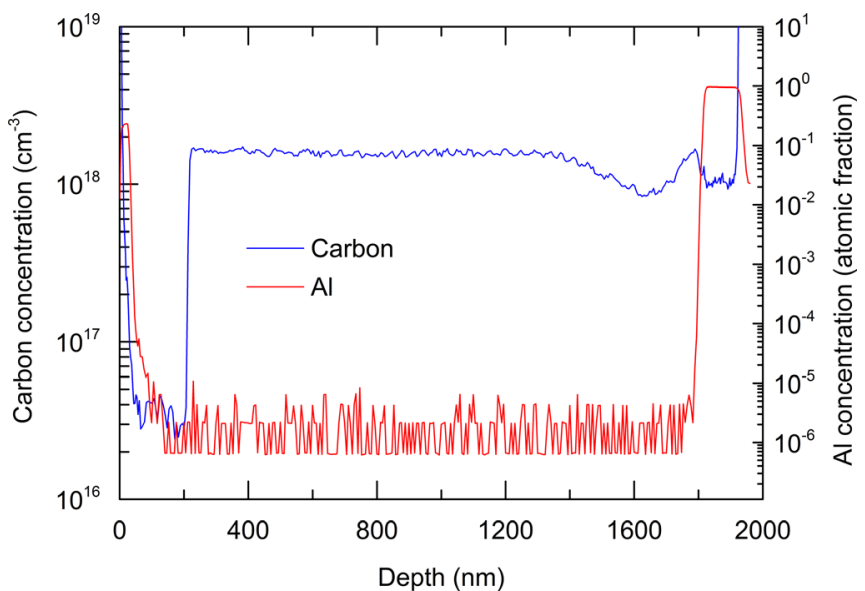

FIG. 3. The carbon incorporation in epitaxial GaN using propane as a dopant gas and aluminum incorporation in $\mathrm{AlN}$ nucleation and $\mathrm{AlGaN}$ barrier layer as measured by SIMS.

use of a gaseous carbon source even for very high carbon doping. The rocking curve of the AlN (0002) reflection has a FWHM value of 240 arcsec.

The two-stepped carbon doped profile is presented in Fig. 3. There is a slight variation in the carbon incorporation in the beginning of the GaN growth, starting at a depth of $1800 \mathrm{~nm}$. This is most likely due to the temperature stabilization of the growth zone when the growth temperature is changed from the AlN growth temperature of $1170^{\circ} \mathrm{C}$ to the $\mathrm{GaN}$ growth temperature of $1040^{\circ} \mathrm{C}$. Since the growth chamber has a very large thermal mass, the temperature stabilization will take several minutes during this temperature transition, and it is well known that the carbon incorporation increases with decreased temperature. ${ }^{15,16}$ Once the temperature is stabilized, the carbon incorporation shows a flat profile. The carbon doping drops fast down to $\sim 3 \times 10^{16} \mathrm{~cm}^{-3}$ when the propane is removed from the growth zone, which can be seen at a depth of $218 \mathrm{~nm}$. An $\sim 195 \mathrm{~nm}$ undoped GaN layer is then grown followed by the AlGaN/GaN barrier and cap layer $(\sim 26 \mathrm{~nm})$. In Fig. 3, the aluminum concentration is also added to visualize the ending of the AIN nucleation layer and the start of the AlGaN barrier layer. Silicon, oxygen, and hydrogen were below detection limit through the entire epitaxial layer.

The resistivity in the center of the sample measured by the eddy current method is $570 \Omega$ /square. The carrier density is $6.8 \times 10^{12} \mathrm{~cm}^{-2}$, and the mobility is $1626 \mathrm{~cm}^{2} / \mathrm{V} \mathrm{s}$, as obtained from contactless Hall measurements. From CV measurements, the pinch-off voltage is $-2.3 \mathrm{~V}$, and the pinch-off capacitance is $3.07 \mathrm{pF}$, indicating no carriers in the $\mathrm{GaN}$ buffer layer.

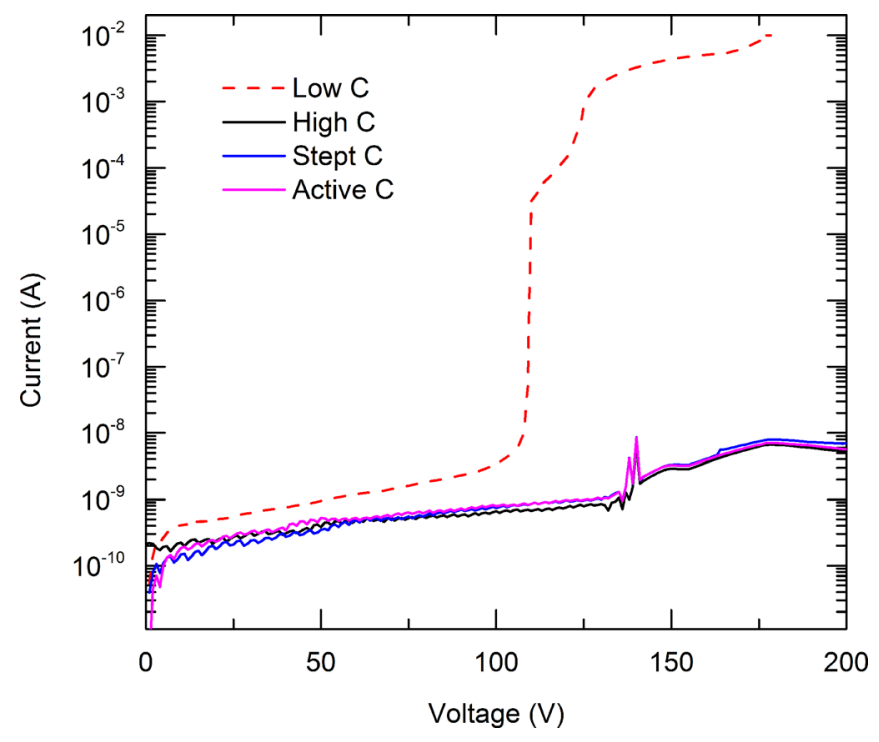

FIG. 4. The leakage current in the GaN buffer layer for the four different samples. "Active C" is the carbon doped structure using propane gas.

The leakage current in the GaN buffer layer was characterized by measuring the current between two mesa-isolated ohmic contacts, separated by $5 \mu \mathrm{m}$. For comparison, three Cdoped structures are included from Ref. 8. These were grown using carbon from the TMGa molecule and are presented together with the structure from this work in Table I.

Although the carbon doped sample using propane gas has a slightly different doping profile compared to the three structures presented in Table I, it closely resembles the "stepped C" structure. For all structures except "Low C," the leakage current was below $1 \mathrm{nA}$. It is clear that the propane doping suppresses buffer leakage, and that the effect is comparable to the growth tuning process (see Fig. 4).

The sheet resistance $\left(\mathrm{R}_{\mathrm{sh}}\right)$, the electron mobility $(\mu)$, and the sheet carrier density $\left(\mathrm{n}_{\mathrm{s}}\right)$ in the 2DEG was measured before and after processing (see Table II). The low and the high carbon structure (Low:C, High:C) had a $2 \mathrm{~nm}$ AlN exclusion layer followed by a $18 \mathrm{~nm} \mathrm{Al}_{0.25} \mathrm{Ga}_{0.75} \mathrm{~N}$ barrier layer. The stepped carbon structure (Stepped:C) had a $2 \mathrm{~nm}$ AlN exclusion layer, a $15 \mathrm{~nm} \mathrm{Al}_{0.30} \mathrm{Ga}_{0.70} \mathrm{~N}$ barrier layer, and a $3 \mathrm{~nm}$ GaN cap layer. The barrier structures differ between the samples and hence the electron mobility and carrier density is not directly comparable. However, the mobility of the active carbon doped structure (Active:C) is in the same order as the other three samples, using carbon from the TMGa molecule, i.e., there is no sign that the propane doping significantly degrade the 2DEG. The lower sheet carrier density in the Active:C structure is related to the lower aluminum content in the $\mathrm{AlGaN}$ barrier layer.

TABLE I. Carbon doped structures using carbon from the TMGa precursor and carbon from propane gas (Active:C). Layer 1 is closest to the AlN nucleation layer.

\begin{tabular}{lcccc}
\hline \hline Reference & Name & $\begin{array}{c}\text { Layer thickness 1, } \\
\text { C-doping concentration }\end{array}$ & $\begin{array}{c}\text { Layer thickness 2, } \\
\text { C-doping concentration }\end{array}$ & $\begin{array}{c}\text { Layer thickness 3, } \\
\text { C-doping concentration }\end{array}$ \\
\hline 8 & High:C & $1800 \mathrm{~nm}, 5 \times 10^{17} \mathrm{~cm}^{-3}$ & & \\
8 & Low:C & $1800 \mathrm{~nm}, 1 \times 10^{16} \mathrm{~cm}^{-3}$ & & $200 \mathrm{~nm}, 1 \times 10^{16} \mathrm{~cm}^{-3}$ \\
8 & Stepped:C & $1500 \mathrm{~nm}, 1 \times 10^{18} \mathrm{~cm}^{-3}$ & $100 \mathrm{~nm}, 1 \times 10^{17} \mathrm{~cm}^{-3}$ & $195 \mathrm{~nm}, 4 \times 10^{16} \mathrm{~cm}^{-3}$ \\
This work & Active:C & $1780 \mathrm{~nm}, 1.5 \times 10^{18} \mathrm{~cm}^{-3}$ & & \\
\hline \hline
\end{tabular}


TABLE II. Before processing, the sheet resistance $\left(\mathrm{R}_{\mathrm{sh}}\right)$ was measured using an eddy-current technique and the mobility $(\mu)$ and the sheet carrier density $\left(\mathrm{n}_{\mathrm{s}}\right)$ were measured using a contactless technique from Lehighton. After processing, the same parameters were extracted from Hall measurements. High:C, Low:C, and Stepped:C data before and after processing are from Ref. 8 .

\begin{tabular}{|c|c|c|c|c|c|}
\hline & Reference & Material & $\mathrm{R}_{\mathrm{s}} \mathrm{h}(\Omega / \mathrm{sq})$ & $\mu\left(\mathrm{cm}^{2} / \mathrm{V} \mathrm{s}\right)$ & $\mathrm{n}_{\mathrm{s}}\left(\times 10^{13} \mathrm{~cm}^{-2}\right)$ \\
\hline \multirow[t]{4}{*}{ Before processing } & 8 & High:C & 373 & 1550 & 1.10 \\
\hline & 8 & Low:C & 270 & 1960 & 1.21 \\
\hline & 8 & Stepped:C & 356 & 1740 & 1.00 \\
\hline & This work & Active:C & 570 & 1630 & 0.68 \\
\hline \multirow[t]{4}{*}{ After processing } & 8 & High:C & $373-437$ & $1470-1730$ & 0.97 \\
\hline & 8 & Low:C & 272 & 1980 & 1.16 \\
\hline & 8 & Stepped:C & 305 & 1640 & 1.25 \\
\hline & This work & Active:C & 569 & 1840 & 0.59 \\
\hline
\end{tabular}
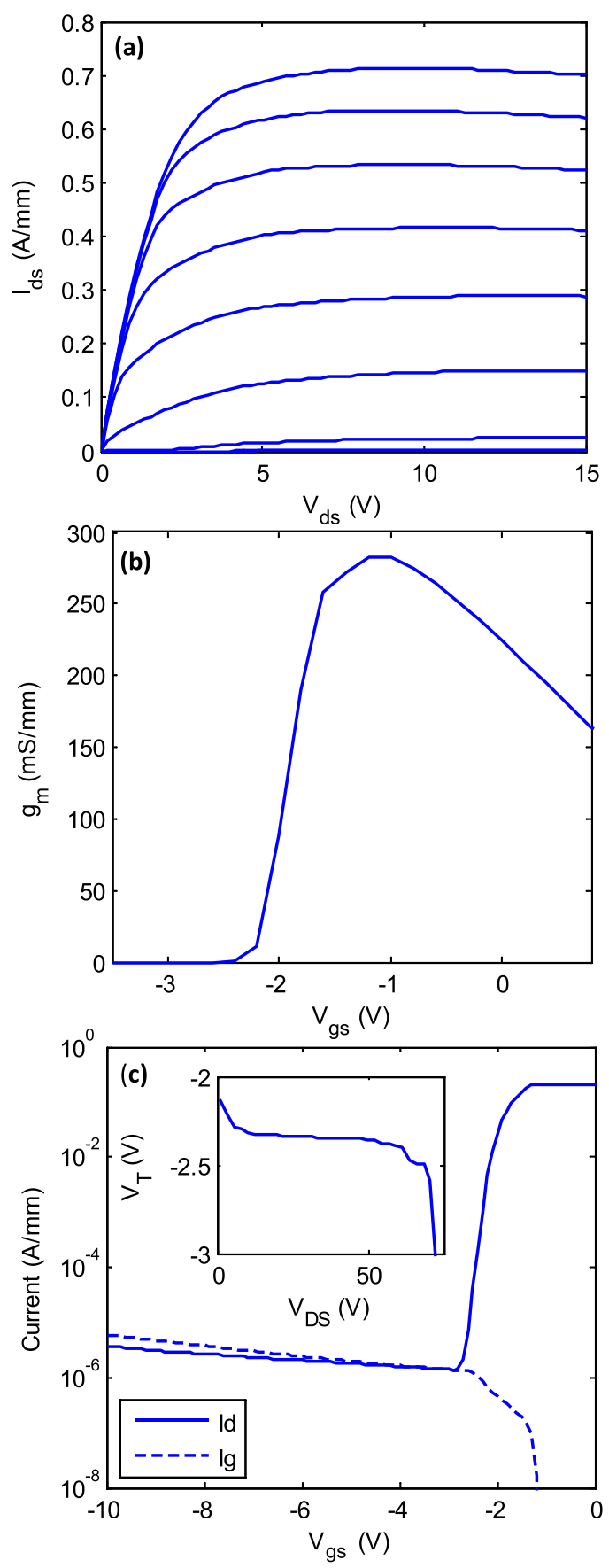

FIG. 5. DC characteristics of the Active:C sample with a gate length of 0.2 $\mu \mathrm{m}$. (a) Drain current versus drain voltage for $\mathrm{V}_{\mathrm{GS}}=-3: 0.5: 1 \mathrm{~V}$. (b) $\mathrm{g}_{\mathrm{m}}$ versus gate voltage for $V_{D S}=10 \mathrm{~V}$. (c) Drain and gate currents at $V_{D S}=10 \mathrm{~V}$ as a function of gate voltage. Threshold voltage versus drain voltage (inset).
The IV characteristics of the Active: $\mathrm{C}$ sample are shown in Fig. 5. The maximum current is $0.7 \mathrm{~A} / \mathrm{mm}$ at $\mathrm{V}_{\mathrm{GS}}=1 \mathrm{~V}$. A slight decrease in current is seen at high currents and voltages due to self-heating effects. $\mathrm{R}_{\text {on }}$ is extracted to $2.6 \Omega$ $\mathrm{mm}$ and is mainly influenced by the large $\mathrm{R}_{\mathrm{sh}}$. Peak transconductance $\left(\mathrm{g}_{\mathrm{m}}\right)$ is around $300 \mathrm{mS} / \mathrm{mm}$. The low leakage currents, below $2 \times 10^{-6} \mathrm{~A} / \mathrm{mm}$, are measured at $\mathrm{V}_{\mathrm{DS}}=10 \mathrm{~V}$. The drain-induced barrier lowering (DIBL) is a measure of how the threshold voltage $\left(\mathrm{V}_{\mathrm{T}}\right)$ changes with applied drain bias and is used as an estimate of buffer isolation. In the range of $10 \mathrm{~V}<\mathrm{V}_{\mathrm{DS}}<60 \mathrm{~V}, \mathrm{~V}_{\mathrm{T}}$ is found to be more or less constant, inset Fig. 5(c). At $\mathrm{V}_{\mathrm{DS}}=30 \mathrm{~V}$, a DIBL of $0.1 \mathrm{mV} /$ $\mathrm{V}$ is extracted. This extremely low value is made possible by the good isolation in the buffer. At the same $V_{D S}$, the subthreshold slope (SS) was measured to $140 \mathrm{mV} / \mathrm{dec}$.

In conclusion, a carbon doped GaN buffer layer in a HEMT heterostructure has been grown using propane as carbon source. The electrical and physical properties of the structure were characterized, and HEMTs were processed to evaluate isolation properties. No memory effect can be seen using propane as dopant gas, and the carbon incorporation drops almost two orders of magnitude over a thickness of $\sim 25 \mathrm{~nm}$, allowing for the design of very sharp doping profiles. This could be achieved without changing temperature, pressure, or III-V ratio. The high carbon doping did not result in any structural or morphological breakdown, and the $\omega$ rocking curve of the (002) and (102) reflection plane showed very narrow peaks. The processed device has a very low leakage current and a very high resistivity in the buffer layer, similar to carbon doped structures using carbon from the TMGa. No major difference in electron mobility could be seen between the different doped structures. Growth of abrupt carbon doping profile without sacrificing the quality of the GaN epilayer facilitates design of buffer doping profiles to optimize GaN HEMT characteristics, e.g., low leakage, high breakdown voltage, and low dispersive effects. We have demonstrated that a carbon doped GaN buffer using a gaseous source may be an attractive alternative to iron doping for improved device properties.

The authors would like to acknowledge the funding support from the Swedish Defense Materiel Administration (FMV) and the Swedish Foundation for Strategic Research (SSF). Jr-Tai Chen is also acknowledged for the growth of the three carbon doped structures using carbon from the TMGa molecule. 
${ }^{1}$ S. C. Binari, P. B. Klein, and T. E. Kazior, Proc. IEEE 90, 1048-1058 (2002). ${ }^{2}$ U. K. Mishra, L. Shen, T. E. Kazior, and Y.-F. Wu, Proc. IEEE 96, 287-305 (2008).

${ }^{3}$ Y. F. Wu, A. Saxler, M. Moore, R. P. Smith, S. Sheppard, T. Chavarkar, T. Wisleder, U. K. Mishra, and P. Parikh, IEEE Electron Device Lett. 25, 117-119 (2004).

${ }^{4}$ S. Heikman, S. Keller, S. P. DenBaars, and U. K. Mishra, Appl. Phys. Lett. 81, 439 (2002).

${ }^{5}$ V. Desmaris, M. Rudzinski, N. Rorsman, P. R. Hageman, P. K. Larsen, H. Zirath, T. C. Rodle, and H. F. F. Jos, IEEE Trans. Electron Devices 53, 2413-2417 (2006).

${ }^{6}$ E. Bahat-Treidel, F. Brunner, O. Hilt, E. Cho, J. Wurfl, and G. Tränkle, IEEE Trans. Electron Devices 57, 3050-3058 (2010).

${ }^{7}$ J. Chen, U. Forsberg, and E. Janzén, Appl. Phys. Lett. 102, 193506 (2013). ${ }^{8}$ S. Gustafsson, J. T. Chen, J. Bergsten, U. Forsberg, M. Thorsell, E. Janzen, and N. Rorsman, IEEE Trans. Electron Devices 62, 2162-2169 (2015).
${ }^{9}$ X. Li, Ö. Danielsson, H. Pedersen, E. Janzén, and U. Forsberg, J. Vac. Sci. Technol., B 33, 021208 (2015).

${ }^{10}$ U. Forsberg, A. Lundskog, A. Kakanakova-Georgieva, R. Ciechonski, and E. Janzén, J. Cryst. Growth 311, 3007 (2009).

${ }^{11}$ A. Kakanakova-Georgieva, U. Forsberg, I. G. Ivanov, and E. Janzén, J. Cryst. Growth 300, 100 (2007).

${ }^{12}$ M. Fagerlind and N. Rorsman, Phys. Status Solidi C 8, 2204 (2011).

${ }^{13}$ A. Malmros, H. Blanck, and N. Rorsman, Semicond. Sci. Technol. 26, 75006 (2011).

${ }^{14}$ J. Bergsten, A. Malmros, M. Tordjman, P. Gamarra, C. Lacam, M.-A. di Forte-Poisson, and N. Rorsman, Semicond. Sci. Technol. 30, 105034 (2015).

${ }^{15}$ A. Ishibashi, H. Takeishi, M. Mannoh, Y. Yabuuchi, and Y. Ban, J. Electron. Mater. 25, 799 (1996).

${ }^{16}$ G. Parish, S. Keller, S. P. Denbaars, and U. K. Mishra, J. Electron. Mater. 29, 15 (2000). 